\title{
An Improved Artificial Fish Swarm Algorithm
}

\author{
Jianshen Peng ${ }^{(1),(2)}, Z_{\text {Zhenwu Wan(3), Qingjin Wei(1), Hui Jiang }}^{(2)}$, Shixiong Wei(1) \\ (1) Colleges of Physics and Mechanical \& Electronic Engineering, Hechi University, Yizhou, CHINA \\ (2) Colleges and Universities Key Laboratory of Intelligent Integrated Automation, Guilin University of Electronic Technology, \\ Guilin, CHINA \\ (3) Department of Information Engineering, Huaxia College, Wuhan University of Technology, Wuhan, CHINA
}

\section{SUMMARY}

The purpose of this paper is to improve the performance of the original AFSA algorithm at the optimal accuracy rate and overcome the weakness of the algorithm which is also trapped in the local optimum. To this end, the original AFSA was further improved based on the tabu strategy. Specifically, the reproduction and death were introduced to protect the best individuals and eliminate poor quality fish, so as to increase convergence and accuracy. Through simulation, it is proved that our solution can achieve high accuracy, good global convergence, and strong resistance to local minimum. The findings bring new light on the application of AFSA and provide valuable reference to studies in related fields.

KEY WORDS: AFSA; tabu strategy; free domain; reproduction and death; swarm intelligence.

\section{INTRODUCTION}

Artificial life, a mimicry of natural living systems, has become a popular trend in multidisciplinary research and development. Inspired by the phenomenon that animals of similar size tend to aggregate together, numerous swarm intelligence optimization algorithms have been developed based on the bionics theory. Unfortunately, all these algorithms have their respective drawbacks. For instance, the ant colony algorithm often consumes too much time and falls into the local optimum trap; the particle swarm algorithm [1] is low in accuracy and easy to run into local optimum, too; the firefly algorithm [2] suffers from poor convergence and local optimum trap.

Considering that fish prefer to live in nutrient-rich regions, Li Xiaolei created an intelligent optimization algorithm called the artificial fish swarm algorithm (AFSA) through the simulation of fish foraging behaviour with computer animation technologies [3-7]. AFSA is a classic animal behaviour-based artificial intelligence application capable of avoiding local extremes, making adaptive optimization and realizing fast convergence. The algorithm exhibitshigh robustness and only needs to compare the pros and cons of the objective function valuewithdetermining the specific initial values. AFSA pioneers the highly nonlinear solution to multidimensional planning problems. Over the years, it has been successfully applied in grid 
reconfiguration, noise reduction and multi-user detection, etc. However, the algorithm also has many shortcomings, such as strong blindness, poor optimization, trapping in local optimum, and low convergence efficiency.

To overcome such shortcomings of AFSA, various solutions have been proposed by scholars at home and abroad. For example, Shen et al. treated stagnant artificial fish with a differential evolution policy and succeeded in preventing local optimum and enhancing the convergence efficiency [8]. Duan and Zhou improved the AFSA performance by optimizing the algorithm structure into that of the hybrid particle swarm optimization algorithm [9]. Ou and Zhou introduced the fluorescence factor of the firefly algorithm into AFSA and set up adaptive steps to improve convergence speed and accuracy [10]. Ma and Liu modified AFSA with an adaptive vision, and thus reduced its computing load and enhanced its accuracy [11]. Fan et al. integrated foods, clusters, rear-ends and controls into the search field method, aiming to elevate the hit rate, efficiency and convergence [12]. To boost the performance of the algorithm, Peng altered the mutation operator policy, adopted the adaptive step length, and improved the visual field. Peng improved the foraging behaviour to enhance the convergence rate of AFSA [13]. Ouyang and Zhou et al. [14] introduced the fluorescence factor of the firefly algorithm to improve the convergence speed and accuracy; Chen et al. [15] changed the mutation operator policy and improved the adaptive step size and the visual field to enhance the algorithm performance; Wang et al. [16] improved the foraging behaviour in the artificial fish swarm algorithm to enhance the convergence rate and so on.

To some extents, the above methods have indeed improved the performance of AFSA. However, the convergence efficiency and the optimization quality are still not satisfactory. Therefore, this paper makes further improvements based on the tabu strategy to overcome local optimum and speed up convergence. Specifically, the reproduction and death are introduced to protect the best individuals and eliminate the low-quality fish, so as to increase convergence and accuracy. Through simulation, it is proved that our solution can achieve high accuracy, good global convergence, and strong resistance to local minimum.

\section{AFSA}

In water bodies, fish tend to follow the swarm in food foraging. Sometimes, the fish swarm may move to faraway places to look for food, so nutrient-rich waters naturally have more fish. In general, the number of fish in an area is positively related to the concentration of nutrients.

Table 1 Information of Datasets

\begin{tabular}{cc}
\hline Parameter name & Mathematical function expression \\
\hline Artificial fish individual state quantity & $X=\left(x_{1}, x_{2}, \ldots, x_{n}\right)$ \\
Artificial fish desire variables & $X_{i}(i=1,2, \ldots, i)$ \\
Food concentration of the artificial fish current location & $Y_{i}$ \\
The objective function & $Y=f(x)$ \\
Distance between artificial individual fish & $d_{i, j}=\left\|x_{i}-X_{j}\right\|$ \\
Sensing range artificial fish & visual \\
Steps & step \\
Crowding factor & $\delta \delta$ \\
The maximum number of artificial fish temptations each iteration & trynumber
\end{tabular}

Note: This paper only discusses the maximization problem. The minimization problem, the exact opposite to the maximization problem, was omitted here. 
Inspired by this fishswarming behaviour, AFSA was created to integrate individual information through target process optimization. Table 1 lists the specific steps of AFSA.

\subsection{FORAGING BEHAVIOUR}

Suppose that the desired state and the desired food concentration of artificial fish $i$ are $X_{i}$ and $Y_{i}$, respectively. First, obtain a random state $X_{j}$ and a food concentration $Y_{j}$ in its field of vision. If $Y_{j}$ is greater than the desired concentration $Y_{i}$, move to the next step "swarming behaviour"; or otherwise, find a random state $X_{j}$ and a food concentration $Y_{j}$ in the field of vision again, and examine if the new food concentration meets the demand. If the desired state is not reached after a number of rounds, go to the step "random behaviour".

\subsection{SWARMING BEHAVIOUR}

After artificial fish $i$ reach the desired state $X_{i}$, count the total number of fishnf within its field of vision ( $d_{i j}<$ visual). If $n f=0$, return to the step "foraging behaviour"; or otherwise, find the centre position $X_{c}$ within the field of vision, and calculate the corresponding position of the objective function value $Y_{c}$. If the food concentration at the centre position exceeds the desired food concentration $\left(Y_{i}<Y_{c} \& \& \frac{Y_{c}}{n f}>\delta Y_{i}\right)$, move to the direction of the state $X_{c}$; or otherwise, return to the step "foraging behaviour".

\subsection{REAR-END BEHAVIOUR}

Suppose that artificial fish i have reached the desired state $X_{i}$, count the number of partners $n f$ in the neighbourhood ( $d_{i j}<$ visual). If $n f=0$, return to the step "foraging behaviour"; or otherwise, calculate the maximum number of partners $X_{\max }$ and the highest food concentration $Y_{\max }$, and determine the optimal partner position which is rich in food and less crowded $\left(Y_{i}<Y_{c} \& \& \frac{Y_{c}}{n f}>\delta Y_{i}\right)$. If the optimal partner position is reached, move to the next step; or otherwise, return to the step "foraging behaviour".

\subsection{RANDOM BEHAVIOUR}

With foraging behaviour as the default, artificial fish i get a random state $X_{j}$ in its field of vision (1) Behaviour evaluation.

Evaluate the impact of the current situation on problem-solving, and select the optimal option based on the conclusion. First, compare the results of swarming behaviour and rear-end behaviour, and execute the better one. The foraging behaviour is the default behaviour.

\subsection{BULLETIN BOARD}

The bulletin board is provided to record and display the optimization results of each step, and to verify if the results are better than those of the previous steps. 
Overall, the foraging behaviour setsthe basis for convergence; the swarming behaviour ensures the convergence stability; the rear-end behaviour enhances the speed and global nature of convergence; the behaviour evaluation safeguards the efficiency and stability of AFSA; and the bulletin board guarantees the quality of the optimization results.

\section{IMPROVED AFSA}

\subsection{ADAPTIVE STEP LENGTH AND FIELD OF VIEW BASED ON THE TABU STRATEGY}

Following the tabu strategy, set up a dynamic tabu domain $U_{j}$ and free domain $U_{z}$ of artificial fish within the search field $U$ (See Eq. (1) for the relationship between these three parameters). Keep updating the step length until the steps on the bulletin board $p$ reaches $d$, and determine the flag pole partial range $U_{d}$. By Eqs. (2) and (3), adjust the search tabus that ban artificial fish from entering thearea, thus changing the number of artificial fish moving into the free domain. The step length and field of view should be altered adaptively by Eqs. (4) and (5),

$$
\begin{gathered}
U=U_{z}+U_{i} \\
U_{z} \in[a, b] \\
U_{j}=U-U_{z} \\
\text { step }=\alpha \times(\text { abs }(b-a) \div \text { fishmum }) \\
\text { vissual }=\beta \times \text { step }
\end{gathered}
$$

where $a$ is the absolute value of the horizontal position of the optimal artificial fish and $b$ is that of the vertical position of the worst artificial fish; the two positions are combined into the optimal position of artificial fish. $\alpha$ and $\beta$ are variable factors. The tabu strategy overcomes the local optimum trap, and expands the scope of foraging, leading to higher convergence rate and optimization accuracy. Based on the tabu strategy, the adaptive adjustment of the step length and the field of view brings the initial values to more appropriate levels. These moves strike a balance between global and local search capabilities, speed up the operation, and ensure the computing accuracy.

\subsection{REPRODUCTION AND DEATH}

Reproduction and death are the nature of all living creatures. Introducing the two concepts to AFSA improves the similarity between artificial fish and natural one, and reflects the idea of artificial intelligence. In order to survive, fish must find enough amount of food to meet their basic needs. Such amount is called the survival amount. Each foraging behaviour may end up with different amounts of food, depending on the length of travel and difficulty in foraging. If the amount of food exceeds the survival amount, the excess portion will be stored for the fish to grow up. The amount of stored food is positively correlated with the vitality of the fish. The greater the amount of food stored, the more likely is for the fish to reproduce. If the amount of food falls short of the survival amount, the fish will not be able to sustain their life and will face death. Hence, food and fitness are selected as the feature factors ofthe reproduction and death of artificial fish. 
Food-based method: Let $Y_{s}$ be the survival amount, and $Y_{i}$ be the amount of food found in each step. After optimization, if $Y_{i}$ is smaller than $Y_{S}$, the artificial fish will be eliminated; if it is greater than $Y_{S}$, most artificial fish will give birth to new fish. The relationship between the number of new fish and the number of dead fish is expressed in Eq. (6). If $Y_{i}$ is equal to $Y_{S}$, go to the step of "random behaviour".

$$
X_{\text {new }}=X_{\text {max }}+\text { Rand } \times \text { step }
$$

where Rand $\in(0,1)$.

This method is simple and flexible, and applicable to various environments. As long as the food factor is available, the method can be used without any special parameter in Figure 1.

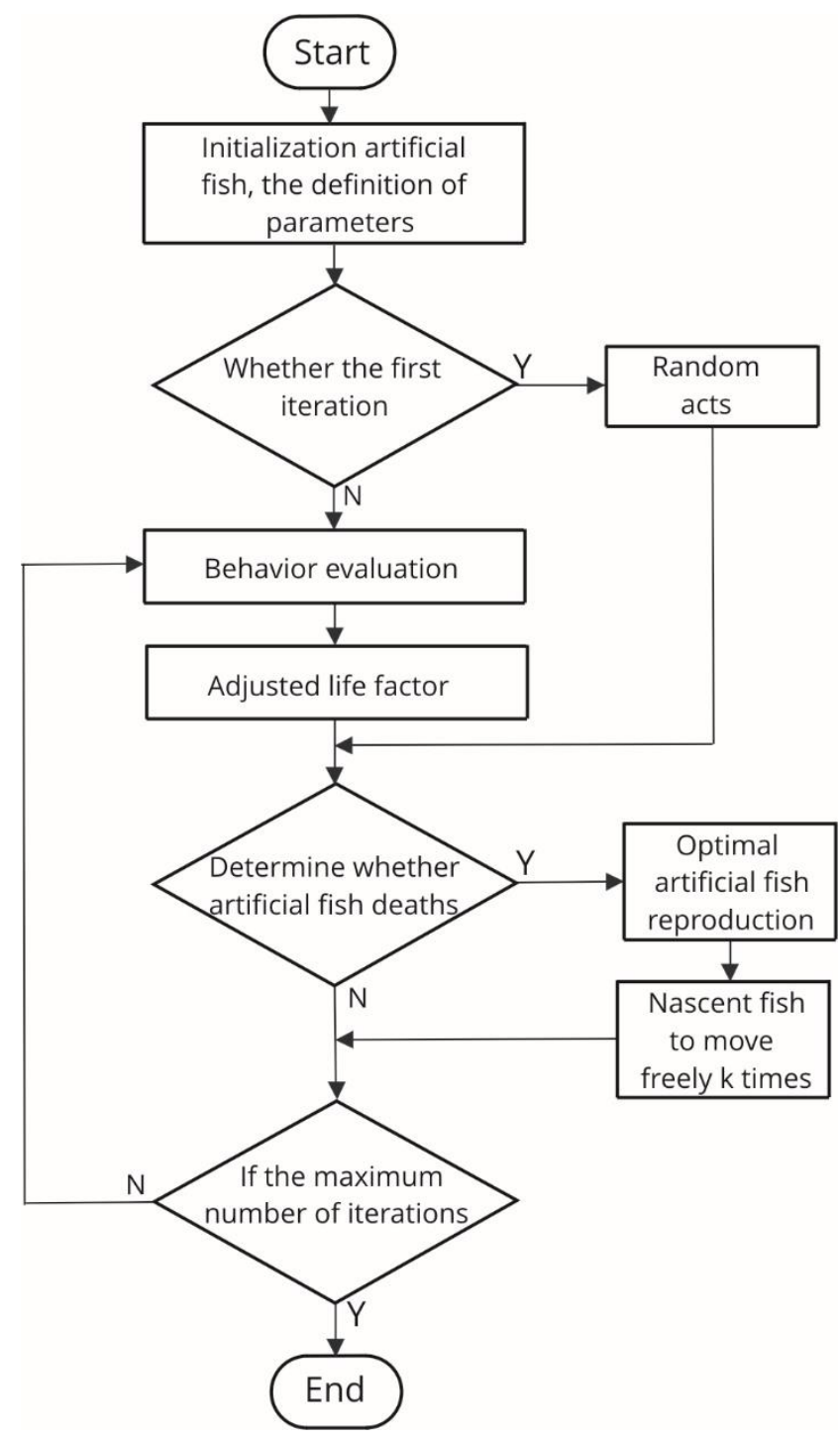

Fig. 1 Reproduction and death I algorithm flowchart 1

Fitness-based method: As mentioned above, the excess portion of food will be stored for the fish to grow up. In other words, the amount of food can be demonstrated by the fitness of the artificial fish. Let the number of artificial fish be fishnum, the survival amount be survival_food, the amount of food found in each step $Y_{i}$, and the desired survival amount in each step $b$. Based on the amount of food consumed, the fitness of artificial fish is obtained by Eq. (8). The food 
concentrations are ranked in ascending order. The higher the concentration, the greater the fitness or the chance for the artificial fish to survive is. If the fitness is below 0 , the artificial fish will die; and if the fitness is greater than 0 , most artificial fish will give birth to new fish. The number of new fish will offset the number of dead fish; and if the fitness equals 0 , go to the step of "random behaviour".

$$
\begin{aligned}
& c=i-k \\
& \text { survival food }(j)=\operatorname{survival} f o o d(j)+c
\end{aligned}
$$

where $i$ is the food concentration for the $j$-th fish in a step; $k$ is the step number (fishnum/2), rounded to the nearest integer.

The flow chart of the improved AFSA is shown in Figure 2.

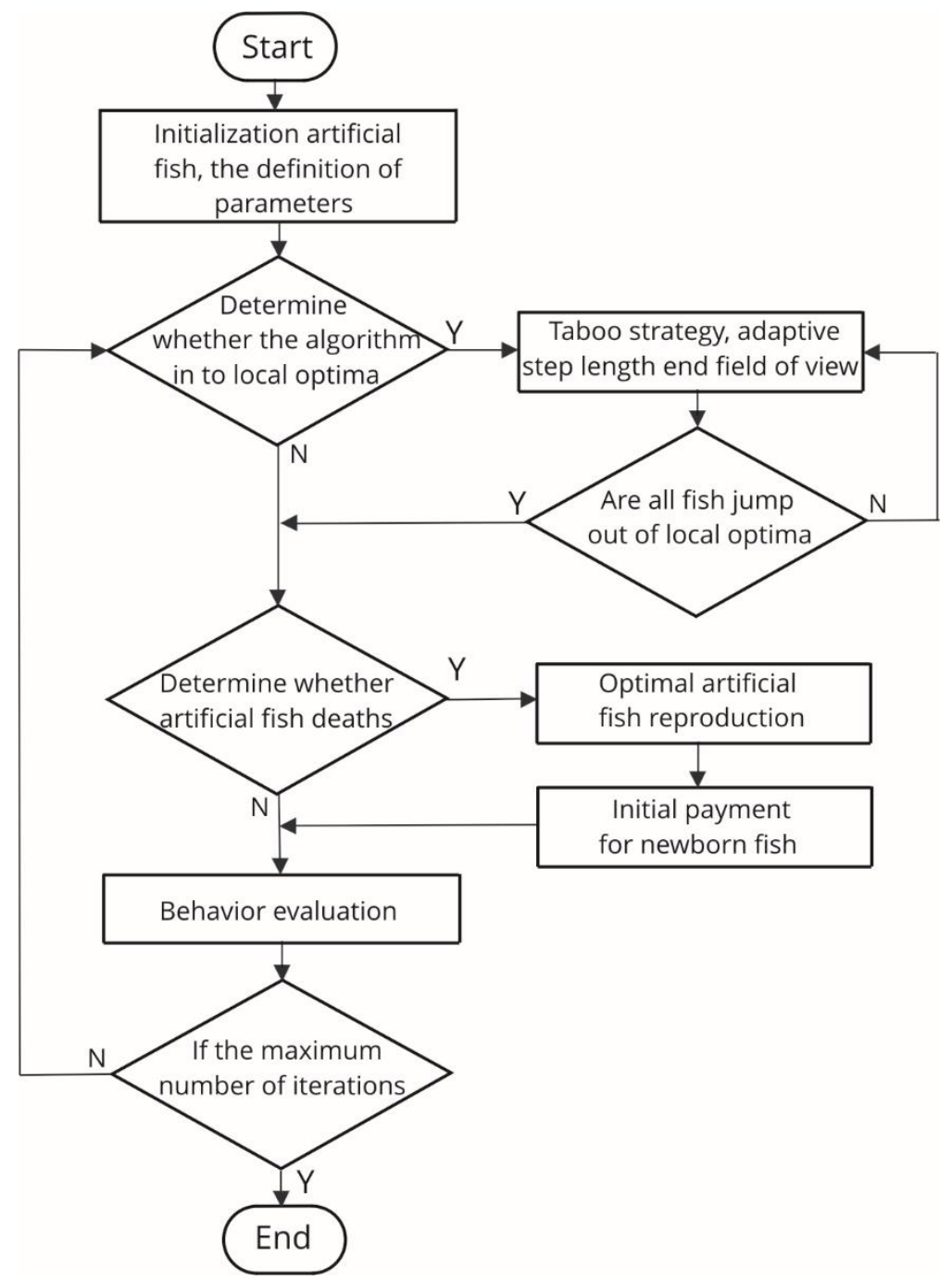

Fig. 2 Improved AFSA flowchart

Steps of the improved-AFSA

- Step 1: Initialize the fish swarm and the parameters.

- Step 2: Execute the foraging, swarming, rear-end and random behaviours, and select the optimal behaviour. 
- Step 3: Check if the algorithm falls into the local optimum; if so, delineate the tabu domain and the free domain, and adjust the step length and field of view to jump out of the local optimum trap.

- Step 4: Introduce reproduction and death to the artificial fish swarm.

- Step 5: If the termination condition is satisfied, terminate the algorithm; otherwise, return to Step 2.

\section{SIMULATION ANALYSIS}

With the aid of Matlab 2010a, the simulation analysis is performed on the test function (Figure 3), [17]:

F1: $f(x, y)=\frac{\sin (x)}{x} \frac{\sin (y)}{y}$, where $|x| \leq 10$ and $|y| \leq 10$. There are many extreme points around the optimal solution at the position $(0,0)$. The optimal value is 1 .

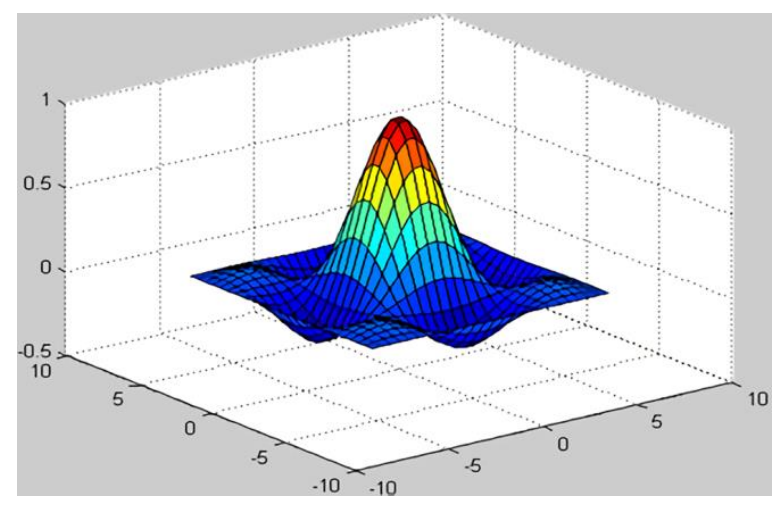

Fig. 3 Function F1 mesh Figure

The parameters are configured as follows: fishnum $=10$, number of steps $=100$, field of view $=1$, step length $=0.1$, average crowding factor $=0.618$, and maximum number of steps MAXGEN $=50$.

To fully demonstrate the effects of improvements, several improved methods are compared with the original AFSA, namely the AFSA improved by the food-based method (AFSA-SSI), the one improved by the tabu strategy (AFSA-J), the one improved by the fitness-based method (AFSA-SSII), and the one improved jointly by the tabu strategy and the fitness-based method (AFSA-J-SSII). Table 2 shows the results of the five algorithms.

Table 2 Five different algorithms to improve an optimization data comparison function F1

\begin{tabular}{cccc}
\hline Algorithm & Best value & Optimal Solution X1 & Optimal Solution X \\
\hline AFSA & 0.999506328100235 & -0.03533 & 0.04153 \\
AFSA-SS I & 0.999991564767032 & 0.00566 & -0.00413 \\
AFSA-J & 0.999995447275473 & 0.00425 & -0.00312 \\
AFSA-SS II & 0.999997856293427 & -0.00314 & 0.00121 \\
AFSA-J-SS II & 1 & 0 & 0 \\
\hline
\end{tabular}


Through data analysis, it is clear that the four improved algorithms have much better accuracy than the original AFSA. As shown in Figures 4 and 5, the improved AFSA-J-SSII optimal distribution of the position of each fish is more concentrated with AFSA-SSI and AFSA-J, while the original AFSA and AFSA-SSII position distribution is sparse. By comparing the convergence process graphs of each algorithm, it can be seen that the improved AFSA-J-SSII overcomes the local optimal weakness and can achieve global optimization in the further stage.

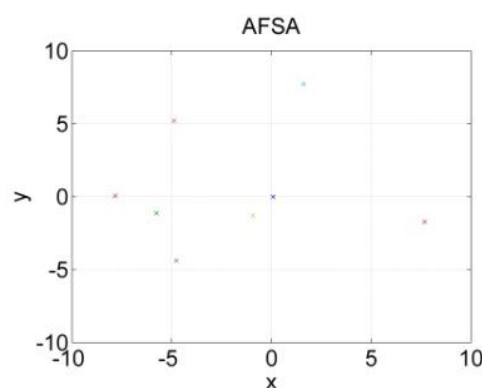

(a) AFSA

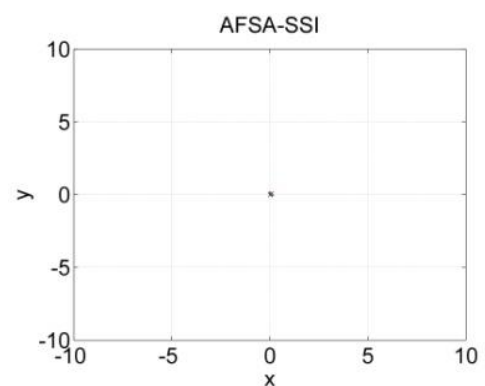

(b) AFSA-SS I

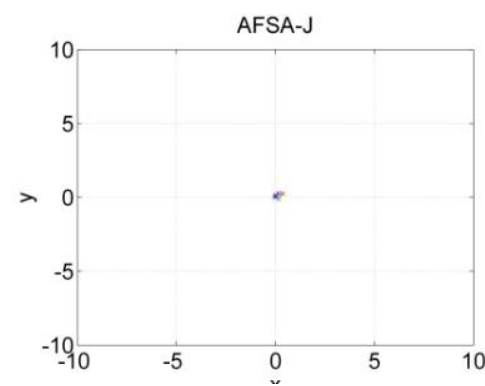

(c) AFSA-J

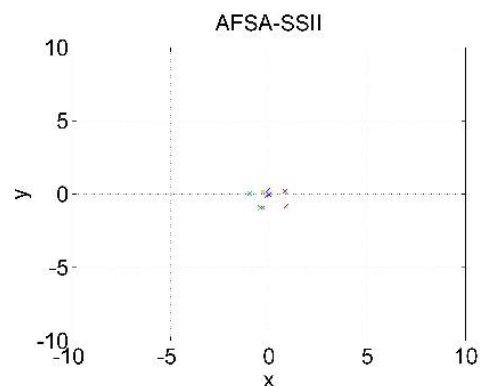

(d) AFSA-SS II

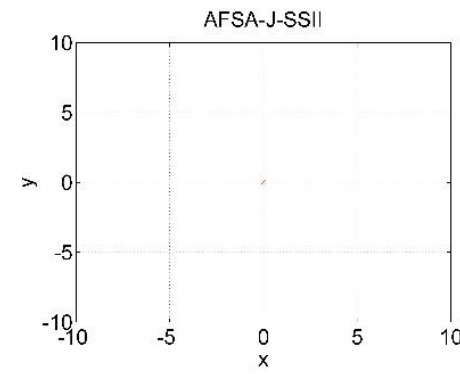

(e) AFSA-J-SS II

Fig. 4 Each fish optimization to location

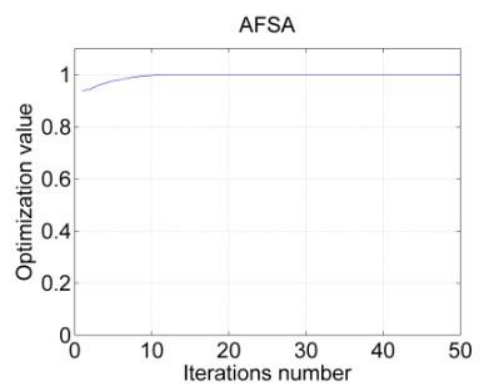

(a) AFSA



(b) AFSA-SS I

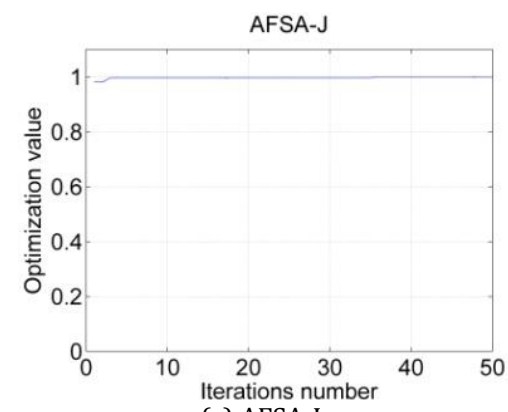

(c) AFSA-J

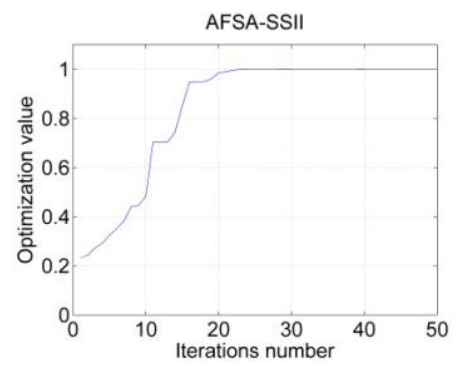

(d) AFSA-SS II

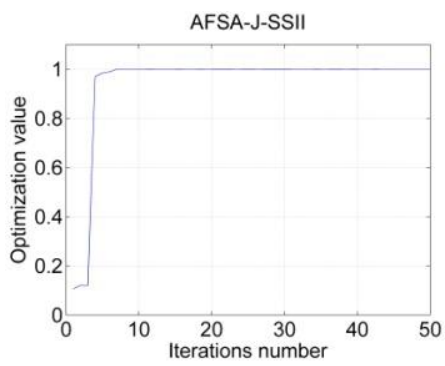

(e) AFSA-J-SS II

Fig. 5 Artificial fish school algorithm iterative processes 
In order to eliminate interferences, the five algorithms are further improved by the same method, and the environment is optimized 100 times in a row. The new results are listed in Table 3. The optimal performance index is $\frac{\left(a_{k}-a^{x}\right)}{a^{*}} 100 \%$, where $a_{k}$ is the runtime, and $a^{*}$ is the theoretical optimal value. The robustness index is $\frac{\left(a_{h}-a^{x}\right)}{a^{*}} 100 \%$, where $a_{h}$ is the average number of steps to obtain the optimal value.

Table 3 Five different improved algorithms optimize data F1 function 100 Comparison

\begin{tabular}{|c|c|c|c|c|c|c|}
\hline \multicolumn{2}{|c|}{ Algorithm Type } & $A F S A$ & AFSA-SS I & $A F S A-J$ & $\begin{array}{l}\text { AFSA-SS } \\
\text { II } \\
\end{array}$ & $\begin{array}{c}\text { AFSA-J-SS } \\
\text { II } \\
\end{array}$ \\
\hline \multirow{4}{*}{ Solutions } & Optimal Solution & 0.99999 & 1.00000 & 1.00000 & 1.00000 & 1 \\
\hline & Worst Solutions & 0.04717 & 0.04719 & 0.99847 & 0.04719 & 1.00000 \\
\hline & $\begin{array}{l}\text { Average optimal } \\
\text { solution }\end{array}$ & 0.70738 & 0.76275 & 0.99994 & 0.71945 & 1.00000 \\
\hline & Best performance & $99.999 \%$ & $100.000 \%$ & $100.000 \%$ & $100.000 \%$ & $100 \%$ \\
\hline \multirow{4}{*}{$\begin{array}{l}\text { Convergence efficiency } \\
\text { (time) }\end{array}$} & Robustness & $70.738 \%$ & $76.275 \%$ & $99.994 \%$ & $71.945 \%$ & $100.000 \%$ \\
\hline & Shortest Time & 0.06240 & 0.09360 & 0.10920 & 0.09360 & 0.34320 \\
\hline & The maximum time & 0.15600 & 0.43680 & 0.23400 & 0.57720 & 0.49920 \\
\hline & Average time & 0.11357 & 0.23104 & 0.14555 & 0.43259 & 0.43181 \\
\hline \multicolumn{2}{|c|}{$\begin{array}{l}\text { Successfully converge to the optimal solution } \\
\text { frequency (>0.999995) }\end{array}$} & 0 & 23 & 50 & 58 & 100 \\
\hline \multicolumn{2}{|c|}{$\begin{array}{l}\text { The number of successful convergences to the } \\
\text { optimal solution }(==1)\end{array}$} & 0 & 0 & 0 & 0 & 74 \\
\hline
\end{tabular}

In Matlab, the disp function can display a figure of six digits after the decimal point. Hence, 0.999995 is adopted as the reference value for this research. According to Table 3, the improved AFSA algorithms still have better accuracy than the original AFSA. For example, AFSA-J-SSII obtains a result greater than 0.999995 at each sub-optimal point by a chance of $100 \%$, while the original AFSA fails to reach this precision, and other algorithm achieves this accuracy about halfonly. In terms of maximum convergence time, the AFSA is comparable to the improved AFSA-J, but the latter can achieve 50 times the number of convergence times to reachthe sub-optimal point. The last thing to note is that AFSA-J-SSII obtains a result equal to 1 at each optimal point by a chance of $74 \%$, while other algorithms fail to achieve this precision. The improved performance and robustness prove the effectiveness of the improved schemes, particularly the superiority of the AFSA-J-SSII algorithm.

To further validate the performance of AFSA-J-SSII, the algorithm is compared with the improved variable step-size adaptive AFSA (IVSSA-AFSA) [18], and the firefly algorithm [19].

The F1 test function is employed for the comparison, and the parameters are set as follows: fishnum $=50$, MAXGEN=100, maximum number of steps $=100$, field of view $=4$, average crowding factor $=0.618$, and step length $=2.5$. For the sake of objectiveness, the results are separated from the algorithms and magnified 20 times before the analysis.

As can be seen from Table 4, AFSA-J-SSII maintains a clear edge over IVSSA-AFSA and the firefly algorithm in both convergence rate and optimization accuracy. AFSA-J-SSII is slightly behind the IVSSA-AFSA in efficiency in the first 40 steps, but soon overtakes the latter in all aspects (e.g. convergence speed, optimization accuracy, and optimal value of F1). The firefly algorithm converges relatively fast at the beginning but ends up with a worse convergence 
efficiency and optimization accuracy than that of IVSSA-AFSA. The results provethe good performance of AFSA-J-SS II.

Table 4 Algorithm, firefly algorithm performance comparison char

\begin{tabular}{cccc}
\hline Iterations & Present algorithm & Literature algorithm & Firefly algorithm \\
\hline 5 & 0.998879235491882 & 0.999735304493828 & 0.986044430428463 \\
10 & 0.999968284463917 & 0.999998979981686 & 0.989230045268146 \\
20 & 0.999999867523749 & 0.999999992061611 & 0.989268080564116 \\
30 & 0.999999998385714 & 0.999999999995475 & 0.990591373374653 \\
40 & 0.999999999999941 & 0.999999999999985 & 0.999553219744618 \\
50 & 1 & 0.999999999999945 & 0.999998329515829 \\
60 & 1 & 0.999999999999931 & 0.999997747621443 \\
70 & 1 & 0.999999999999702 & 0.999983792681562 \\
80 & 1 & 0.999999999999999 & 0.999994362129887 \\
90 & 1 & 0.999999999999982 & 0.999999872354650 \\
100 & 1 & 1.000000000000000 & 0.999999804972407 \\
\hline
\end{tabular}

\section{CONCLUSION}

To overcome the drawbacks of the original AFSA, this paper successfully creates an excellent improved AFSA (AFSA-J-SSII) by introducing the tabu strategy and both reproduction and death into the original one. The simulation results show that AFSA-J-SSII can strike a balance between global and local search capabilities, significantly improving its ability to overcome local minimum and making it superior in adaptive capacity, convergence efficiency and precision. This research introduces a new way for multi-robot path planning and design.

\section{ACKNOWLEDGEMENTS}

The authors are highly thankful to the National Natural Science Foundation of China (Grant No. 61640305), Natural Science Foundation of Guangxi Province (N0.2018GXNSFAA281164). This research was financially supported by the project of outstanding thousand young teachers' training in higher education institutions of Guangxi, Guangxi Colleges and Universities Key Laboratory Breeding Base of System Control and Information Processing, Hechi University research project start-up funds (XJ2015KQ004), Supported by Colleges and Universities Key Laboratory of Intelligent Integrated Automation (GXZDSY2016-04), Research on multi robot cooperative system based on artificial fish swarm algorithm (2017CFC811).

\section{REFERENCES}

[1] N. Li, D.B. Sun, T. Zou, An Analysis for a Particle's Trajectory of PSO Based on Difference Equation, Journal of computer, No. 11, pp. 2052-2060, 2006.

[2] A. Demiriz, K.P. Benefit, M.J.Embrechts, Semi-supervised clustering using genetic algorithm, Proc of Intelligent Engineering Systems through Artificial Neural Networks, No. 3, pp. 809-841, 1999. http://dx.doi.org/10.1080/10255810210623 
[3] X.Y.Tu, Artificial animals for computer animation: Biome-chanics, locomotion, perception, and behavior, ACM Outstanding Ph D Dissertation Bool Series, Springer Verlag, No. 1635, pp. 361-364, 1999.

[4] X.Y. Tu, T. Demetri, Artifical fishes: physics, locomotion, perception, behavior, Computer Graphics \& Interactive Techniques, pp. 43-50, 1994. http://dx.doi.org/10.1145/192161.192170

[5] J. Dean, Animats and what they can tell us, Trends in Cognitive Sciences, No. 2, pp. 60-67, 1998. http://dx.doi.org/10.1016/S1364-6613(98)01120-6

[6] X.L. Li, Z.J. Shao, J.X. Qian, An Optimizing Method Based on Autonomous Animats: Fishswarm Algorithm, Systems Engineering-Theory \& Practice, No. 22, pp. 32-38, 2002. http://dx.doi.org/10.3321/j.issn:1000-6788.2002.11.007

[7] X.L. Li, J.X. Qian, Studies on artificial fish swarm optimization algorithm based on decomposition and coordination techniques, Journal of Circuits and Systems, No. 8, pp. 16, 2003. http://dx.doi.org/10.3969/j.issn.1007-0249.2003.01.001

[8] W. Shen, X. Guo, C. Wu, D. Wu, Forecasting stock indices using radial basis function neural networks optimized by artificial fish swarm algorithm, Knowledge-Based Systems, No. 24, pp. 378-385, 2011. http://dx.doi.org/10.1016/j.knosys.2010.11.001

[9] Q.C. Duan, R.L. Tang, H.Y. Xu, Simulation analysis of the fish swarm algorithm optimized by PSO, Control and Decision, No. 28, pp. 1436-1440, 2011.

[10] Y.Z. Ou, Y.Q. Zhou, Self-adaptive step glowworm swarm optimization algorithm, Journal of Computer Applications, No. 31, pp. 1804-1807. http://dx.doi.org/10.3724/SP.J.1087.2011.01804

[11] X.M. Ma, N. Liu, Improved artificial fish-swarm algorithm based on adaptive vision for solving the shortest path problem, Journal of Communications, No. 35, pp. 1-6, 2014. http://dx.doi.org/10.3969/j.issn.1000-436x.2014.01.001

[12] Y. Fan, D. Wang, M. Sun, Improved Artificial Fish-school Algorithm, Journal of Chongqing Normal University (Natural Science Edition), No. 24, pp. 23-26, 2007. http://dx.doi.org/10.3969/j.issn.1672-6693.2007.03.007

[13] J.S. Peng, The robot path optimization of improved artificial fish-swarm algorithm, Computer Modelling and New Technologies, No. 18, pp. 147-152, 2014.

[14] Z. Ouyang, Y.Q. Zhou, Self-adaptive step glowworm swarm optimization algorithm, Journal of Computer Applications, No. 31, pp. 1804-1807, 2011. http://dx.doi.org/10.3724/SP.J.1087.2011.01804

[15] G.Z. Chen, J.Q. Wang, C.J. Li, X.Y. Lu, An Improved Artificial Fish Swarm Algorithm and Its Applications, Systems Engineering, No. 27, pp. 105-110, 2009.

[16] Z.L. Wang, X.Y. Liu, W.P. Wang, Advanced Artificial Fish Swarm Algorithm, Information Technology and Informatization, No. 10, pp. 46-49, 2010. http://dx.doi.org/10.3969/j.issn.1672-9528.2010.03.13

[17] Y. Li, D.D. Wei, Z. Mu, Z.H. Xiong, Y.H. Wang, W.S. Yin, Study of the time-collocation of signal lamp at intersection, Mathematical Modelling of Engineering Problems, Vol. 2, No. 1, pp. 13-16, 2015. http://dx.doi.org/10.18280/mmep.020104 
[18] R. Azizi, Empirical study of artificial fish swarm algorithm, International Journal of Computing, Communications and Networking, No. 3, pp. 1-7, 2014.

http://dx.doi.org/10.1016/j.esd.2013.09.001

[19] Q.C. Duan, D.W. Huang, L. Lei, Simulation analysis of particle swarm optimization algorithm with extended memory, Control and Decision, No. 26, pp. 1087-1440, 2011. http://dx.doi.org/10.1007/s11518-011-5154-1 